\title{
Quantification of Resource Utilization to Obtain Functional Recovery in Strokes
}

\author{
Kazuaki Kuwabara, ${ }^{a}$, Shinya Matsuda ${ }^{\mathrm{b}}$, Kiyohide Fushimic ${ }^{\mathrm{c}}$, Koichi B. Ishikawa ${ }^{\mathrm{d}}$, \\ Hiromasa Horiguchie, Kenji Fujimori ${ }^{\mathrm{f}}$
}

\begin{abstract}
Background: The increasing elderly population will turn increased attention to the functional recovery and costs for patients with cerebrovascular disease (CVD). Several functional scales like the Barthel index (BI) have been applied in healthcare research and handled as continuous variables for risk adjustment or main outcomes. BI is originally an ordinal variable and we tested whether healthcare resources during acute care settings were utilized evenly among patients with BI improvement, and whether the incremental cost to gain the given BI improvement varied, depending on the functional status at admission.
\end{abstract}

Methods: The study administrative database contained 337,851 CVD patients across 1,050 hospitals from fiscal year (FY) 2006 to 2009 . We identified a total of $61,191 \mathrm{CVD}$ patients with available BI scores among 334 hospitals participating consecutively in the 4 study years. Patient and hospital characteristics, care process, BI score at admission and discharge, length of stay (LOS) and total charges (TC) were analyzed among three BI-at-admission categories. BI at admission was categorized into the following three

Manuscript accepted for publication August 19, 2011

${ }^{\text {a }}$ Graduate School of Medicine, Department of Health Care

Administration and Management, Kyushu University, Fukuoka, Japan

${ }^{\mathrm{b}}$ Department of Preventive Medicine and Community Health, University of Occupational and Environmental Health, Kitakyushu, Japan

${ }^{\mathrm{c}}$ Department of Health Policy and Informatics, Tokyo Medical and

Dental University Graduate School of Medicine, Tokyo, Japan

${ }^{\mathrm{d}}$ Statistics and Cancer Control Division, National Cancer Center, Tokyo, Japan

e Department of Health Management and Policy, the University of Tokyo, Tokyo, Japan

${ }^{\mathrm{f}}$ Division of Medical Management, Graduate School of Medicine Hokkaido University, Sapporo, Japan

${ }^{\mathrm{g}}$ Corresponding author: Kazuaki Kuwabara, Department of Health Care Administration and Management, Graduate School of Medical Sciences, Kyushu University, 3-1-1 Maidashi, Higashi-ku, Fukuoka 812-8582,

Japan. Email: kazu228@basil.ocn.ne.jp

doi:10.4021/jnr42w groups: $<59$ dependent; $60-84$ independent with assistance, and $\geq 85$ nearly completely independent. The effect of a one-point BI gain on LOS and TC was determined using mixed linear regression models.

Results: A total of 22,342 CVD patients across 327 hospitals were observed to have BI improvement at discharge. Among admitted patients, $59.5 \%$ were dependent and $25.6 \%$ were independent with assistance. Dependent status at admission was frequently associated with surgical procedures, ICU accommodation, ventilation and rehabilitation, whereas patients who were independent with assistance only had increased use of ICU and rehabilitation. Better functional status at admission reduced LOS and TC. One-point gain in the BI was associated with shorter LOS in patients with dependent functional status solely, and with more TC in those who were independent with assistance and nearly completely independent.

Conclusions: We observed a variation in incremental cost required for BI improvement among three functional status groups at admission. Policy-makers should be aware of the variation in cost required for a given functional gain depending on the functional status category at admission, and plan the efficient healthcare policy for CVD patients for promoting functional convalescence.

Keywords: Stroke/Cerebrovascular accident; Health policy; Healthcare outcomes; Resource utilization

\section{Introduction}

Modern society is experiencing an aging population. In fiscal year (FY) 2004, approximately $15 \%$ of the Japanese population was at least 70 years of age and accounted for $40.6 \%$ (US\$ 130 billion) of the national healthcare expenditure [1]. As a result, developed countries will have to actively manage costs for patients with chronic illness [2]. Japan must now debate the efficient financing of healthcare delivery along with effective resource allocation because approximately $25 \%$ of the total population will be aged 65 years or older in FY 2025 [1].

This age group is increasingly likely to suffer from such non-communicable diseases as cancer, cerebrovascular disease (CVD) and cardiovascular disease. Disease manage- 
ment is a major focus of research for disease prevention, quality of care in acute care settings, and rehabilitation allocation after acute onset of these illnesses [3-5]. Among them, CVD has been a major cause of death and disability and produces socioeconomic burdens on patients, their families, and on society. Patient characteristics, care process, resource use, and outcomes of mortality, complications, and functional recovery have been investigated to estimate these burdens as well as to establish an effective CVD care system for the targeted population of first-ever stroke, a group expected to obtain full recovery with fewer costs [6-13].

Functional outcomes are evaluated through "activities of daily living" (ADL), expressed by the Barthel index (BI), instrumental ADL, and modified Rankin Score (mRS). These are employed for either risk adjustment or outcome in healthcare service researches [14-16]. Some comparative studies have investigated content or criteria validity of several functional scales through measuring the association or prediction with or among measurements relevant to those scales [14-16]. Other previous researches have only described healthcare cost-adjusted patient case-mixes by use of the BI or the follow-up properties of the BI for CVD patients. To our knowledge, there are few contemporary validity studies on current resource use, though several studies have handled functional scales as continuous variables [6, 13]. These initial studies could contribute to developing efficient cost management for CVD patients in health policy as well as reasonable strategies to stratify or target them according to functional status in healthcare service research.

To measure the variation in current resource use consumed to recover from various stages of functional status at admission, we decided to use the BI, originally an ordinal variable, and restricted the study population to patients observed to achieve functional recovery at discharge. With a Japanese administrative database, we tested whether healthcare resources in acute care settings were utilized evenly, and whether the incremental cost to gain the given BI improvement varied, depending on the functional status at admission expressed by BI.

\section{Methods}

\section{Study settings}

This was a retrospective observational study concurrent with a government-funded research project aiming to develop a Japanese case-mix classification system in cooperation with our research team [17]. The Ministry of Health, Labor and Welfare (MHLW) has been gathering anonymous claims and clinical data across 1,428 hospitals (82 academic hospitals and 1,346 community hospitals) through 2009 . This data, including the date and quantity of patients' daily care processes during hospitalization, were collected by the MHLW and merged into a standardized electronic format for this project over 4 - 6-month periods every year since July 1, FY 2002 (every 4 months from July to October from FY 2002 to 2005 and every 6 months from July to December since FY 2006). A total of $8,010,361$ patients across 1,006 hospitals have voluntarily participated in this research over 4 years since FY 2006. Among them, we analyzed patients with non-traumatic CVD for the degree of functional recovery observed at discharge, who were consecutively treated in 334 hospitals between FY 2006 and 2009, inclusive. Our project was approved by the ethical committee of the University of Occupational and Environmental Health, Fukuoka, Japan.

\section{Study variables}

Study variables included age, sex, principal diagnosis, use of an ambulance, destination (home or another facility), disability status, weighted comorbidity, type of procedure defined in a case-mix definition table, preexisting CVD or atrial fibrillation or flutter, procedure-related complication, deep vein thrombosis and pulmonary embolism (DVT/PE), cognitive impairment, surgical procedures, presence or days of accommodation in the intensive care unit (ICU), administration of ventilation and rehabilitation, hospital ownership and teaching status (academic or community), FY, functional status measured by BI both at admission and at discharge, LOS, and total charges ( 1 US $\$=90$ yen). Age was stratified into four categories of 15 - 64 years, 65 - 74 years, $75-84$ years, and $\geq 85$ years. Emergency admission was defined as transport to hospital by an ambulance. The principal diagnosis of CVD was recorded using the appropriate International Classification of Diseases 10th Revision codes. To explain the variation in CVD subtype, CVD was categorized as transient ischemia (TIA; G45), lacunar status (G46), hemorrhage (I61), subarachnoid hemorrhage (SAH; I60), subdural hematoma (I62), infarction (I63) and reversible ischemic neurological deficits (I65-6) [5, 8]. The MHLW requested the recording of a maximum of four comorbidities and four complications in the database. Weighted comorbidity status was calculated using the Charlson comorbidity index (CCI), which includes CVD, and was examined separately [18]. For complications occurring after admission, DVT/PE, procedure-related complications, and cognitive impairment were examined. Procedure-related complications included wound complications, hematoma, or laceration or disruption of the treated organs by instrumentation or manipulation, for example (T81-T87) [19]. Surgical procedures for CVDs included percutaneous endovascular interventions (e.g. coil implantation, angioplasty, or thrombolysis), carotid endarterectomy, clipping, decompression craniotomy, and evacuation of intracranial hematoma [5]. Hospital ownership was categorized into four groups: national, municipal, private for profit, and non-profit private. In Japan, hospitals have been forbidden to hold stocks or distribute profit for stock hold- 


\begin{tabular}{|c|c|c|c|c|c|}
\hline & & Dependent & $\begin{array}{l}\text { Independent with } \\
\text { assistance }\end{array}$ & $\begin{array}{l}\text { Nearly completely } \\
\text { independent }\end{array}$ & $\mathrm{p}$ \\
\hline Overall & 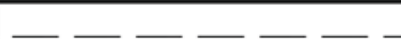 & 13286 & 5722 & 3334 & \\
\hline \multicolumn{6}{|l|}{$\overline{\text { Age }}$} \\
\hline & Mean age $[S D]$ & $70.5[12.6]$ & $69.6[11.9]$ & $68.2[11.9]$ & $<0.001$ \\
\hline & $65-74$ years & $3768(28.4)$ & $1760(30.8)$ & $1126(33.8)$ & \\
\hline & $75-84$ years & $4140(31.2)$ & $1776(31.0)$ & $907(27.2)$ & \\
\hline & 85 - years & $1482(11.2)$ & $441(7.7)$ & $179(5.4)$ & \\
\hline Gender & Male & 7661 (57.7) & $3589(62.7)$ & $2186(65.6)$ & $<0.001$ \\
\hline Destination & not at home & $3897(29.3)$ & $563(9.8)$ & $136(4.1)$ & $<0.001$ \\
\hline Ambulance & use & $6319(47.6)$ & $1561(27.3)$ & $667(20.0)$ & $<0.001$ \\
\hline \multicolumn{6}{|c|}{ Principal disease } \\
\hline & transient ischemia & $893(6.7)$ & $512(8.9)$ & $348(10.4)$ & $<0.001$ \\
\hline & lacunar infarction & $2543(19.1)$ & $1428(25.0)$ & $748(22.4)$ & \\
\hline & SAH & $726(5.5)$ & $69(1.2)$ & $31(0.9)$ & \\
\hline & Hemorhhage & $2010(15.1)$ & $398(7.0)$ & $197(5.9)$ & \\
\hline & Nontraumatic subdural hematoma & $629(4.7)$ & $295(5.2)$ & $169(5.1)$ & \\
\hline & Infarction & $6049(45.5)$ & $2766(48.3)$ & $1562(46.9)$ & \\
\hline & RIND & $436(3.3)$ & $254(4.4)$ & $279(8.4)$ & \\
\hline \multicolumn{2}{|c|}{ Charlson comorbidity index } & & & & $<0.001$ \\
\hline & 1 & $3564(26.8)$ & $1585(27.7)$ & $882(26.5)$ & $<0.001$ \\
\hline & 2 & $2261(17.0)$ & $805(14.1)$ & $422(12.7)$ & \\
\hline & 3 & $824(6.2)$ & $292(5.1)$ & $119(3.6)$ & \\
\hline & 4 & $237(1.8)$ & $73(1.3)$ & $38(1.1)$ & \\
\hline & 5 or more & $71(0.5)$ & $22(0.4)$ & $16(0.5)$ & \\
\hline \multicolumn{2}{|c|}{ Preexisting cerebrovascular disease } & $2173(16.4)$ & $772(13.5)$ & $436(13.1)$ & $<0.001$ \\
\hline \multicolumn{2}{|c|}{ Atrial fibrillation or flutter } & $1009(7.6)$ & $339(5.9)$ & $174(5.2)$ & $<0.001$ \\
\hline \multicolumn{2}{|c|}{ Procedure related complication } & $127(1.0)$ & $14(0.2)$ & $9(0.3)$ & $<0.001$ \\
\hline \multicolumn{2}{|c|}{ Deep vein thrombosis and pulmonary embolism } & $35(0.3)$ & $6(0.1)$ & $4(0.1)$ & 0.043 \\
\hline \multicolumn{2}{|c|}{ Cognitive impairment after admission } & $65(0.5)$ & $10(0.2)$ & $3(0.1)$ & $<0.001$ \\
\hline \multicolumn{6}{|l|}{ Procedure } \\
\hline \multicolumn{6}{|c|}{ Surgical pocedure } \\
\hline & present & $1777(13.4)$ & $492(8.6)$ & $282(8.5)$ & $<0.001$ \\
\hline \multicolumn{6}{|c|}{ Intensive care unit } \\
\hline & administered & $3007(22.6)$ & $612(10.7)$ & $289(8.7)$ & $<0.001$ \\
\hline & mean days administered [SD] & $4.5[4.1]$ & $4.3[3.7]$ & $4.4[3.6]$ & 0.454 \\
\hline \multicolumn{6}{|l|}{ Ventilation } \\
\hline & administered & $180(1.4)$ & $17(0.3)$ & $8(0.2)$ & $<0.001$ \\
\hline & mean days administered [SD] & $4.2[7.3]$ & $1.5[1.2]$ & $1.2[0.4]$ & 0.248 \\
\hline \multicolumn{6}{|c|}{ Rehabilitation } \\
\hline & administered & $9516(71.6)$ & $3574(62.5)$ & $1676(50.3)$ & $<0.001$ \\
\hline & mean days administered [SD] & $19.1[20.1]$ & $13.6[13.3]$ & $11.4[10.1]$ & 0.441 \\
\hline \multicolumn{6}{|c|}{ Hospital ownership } \\
\hline & National & $1003(7.5)$ & $493(8.6)$ & $305(9.1)$ & $<0.001$ \\
\hline & Municipal & $2827(21.3)$ & $1092(19.1)$ & $667(20.0)$ & \\
\hline & Non profit pravate & $4996(37.6)$ & $2099(36.7)$ & $1112(33.4)$ & \\
\hline & Private for profit & $4460(33.6)$ & $2038(35.6)$ & $1250(37.5)$ & \\
\hline \multicolumn{6}{|c|}{ Teaching status } \\
\hline & Academic & $619(4.7)$ & $286(5.0)$ & $176(5.3)$ & 0.266 \\
\hline \multicolumn{6}{|l|}{ Fiscal year } \\
\hline & 2006 & $4010(30.2)$ & $1975(34.5)$ & $1159(34.8)$ & $<0.001$ \\
\hline & 2007 & $3026(22.8)$ & $1302(22.8)$ & $706(21.2)$ & \\
\hline & 2008 & $2610(19.6)$ & $1026(17.9)$ & $654(19.6)$ & \\
\hline & 2009 & $3640(27.4)$ & $1419(24.8)$ & $815(24.4)$ & \\
\hline Outcome an & $\mathrm{d} \cos t$ & & & & \\
\hline Mean $\mathrm{BI}$ at a & dmission, point [SD] & $25.9[20.3]$ & $69.5[7.0]$ & $90.2[4.0]$ & $<0.001$ \\
\hline Mean $\mathrm{Bl}$ at $\mathrm{C}$ & discharge, point [SD] & $79.5[26.8]$ & $95.7[7.9]$ & $99.5[1.9]$ & $<0.001$ \\
\hline Mean Chang & e of $\mathrm{BI}$, point [SD] & $53.7[27.3]$ & $26.3[9.5]$ & $9.3[3.9]$ & $<0.001$ \\
\hline Mean LOS, 0 & days [SD] & $24.8[23.5]$ & $17.3[15.3]$ & $14.3[10.9]$ & $<0.001$ \\
\hline Mean TC, \$ & [SD] & $10826[10061]$ & $7396[6044]$ & 6274 [4724] & $<0.001$ \\
\hline RIND: revers & ible ischemic neurological de & dbarachinoid he & gige. & & \\
\hline $\mathrm{Bl}$; Barthel ir & ndex. LOS; length of hospital stay. & al charge. SD & d deviation & & \\
\hline
\end{tabular}

Figure 1. Patient characteristics and outcomes by functional status at admission. 
ers. Non-profit hospitals are Red Cross and charity hospitals. Academic hospitals included university hospitals and the National Cardiovascular Center involved in educating medical students and postgraduate trainees and in delivering clinical research. Deceased patients were excluded from this analysis.

For functional outcome, disability status was assessed by the BI score at admission and at discharge. BI at admission was categorized into the following three groups: $<59$ dependent; 60 - 84 independent with assistance, and $\geq 85$ nearly completely independent [14]. Changes in BI score were also calculated as $\mathrm{BI}$ at discharge minus $\mathrm{BI}$ at admission; the BI was improved when the change in the BI was greater than zero [6]. We enrolled only the patients with an improved BI score. For cost, TC during hospitalization was examined. In Japan, the delivery of hospital services is determined by a standardized fee-for-service payment system known as the nationally uniform fee table. In this study, TC included physician fees, instrument costs, laboratory or image test costs, and administration fees [5]. TC is considered a good estimate of costs incurred during hospitalization [20].

\section{Statistical analysis}

Proportions and descriptive statistics within each BI-at-admission category were compared using the chi-square test and analysis of variance. A logistic regression model was employed to determine the variation in surgical procedures, ICU admission, ventilation and rehabilitation among the BIat-admission categories.

Mixed-effect models were used to identify the impact of a one-point gain in the BI on LOS and on TC. To control for differences in hospital practices, hospitals were included as a random intercept. Study variables were included as fixed factors in every model. The impact of a one-point gain in the BI on LOS and TC was examined in model 1. Interactions between the three BI-at-admission categories and a one-point gain in the BI were also included (model 2). When explaining the variation in TC, LOS was enrolled as a covariate. All statistical analyses were performed using PASW statistics version 18.0. All P-values are two-tailed and the level of significance was set at $<0.05$.

\section{Results}

The study administrative database originally contained 337,851 CVD patients across 1,050 hospitals from FY 2006 to 2009. Among them, we identified a total of $61,191 \mathrm{CVD}$ patients with available BI scores among 334 hospitals participating consecutively in the 4 study years; 22,342 CVD patients across 327 hospitals were observed to have BI improvement at discharge $(1,081$ patients in 36 academic hospitals and 21,261 patients in 291 community hospitals) and were examined in this study; 13,286 patients $(59.5 \%)$ were dependent and 5,722 (25.6\%) were independent with assistance.

Mean age, proportion of age category, gender, destination, ambulance, principal diagnosis, comorbidity and complication, and hospital ownership were statistically different among the BI-at-admission categories. Proportion of surgical procedures, ICU admission, ventilation, and rehabilitation were significantly different among the BI-at-admission categories, though days of care with the latter three variables were not. Change in BI, LOS and TC were observed to be less with better BI-at-admission category (Fig. 1).

Dependent status at admission was frequently associated with surgical procedures, ICU accommodation, ventilation and rehabilitation, whereas patients who were independent with assistance only had increased use of ICU and rehabilitation. The advanced age categories of 65 years or more were observed to receive more surgical procedures and less rehabilitation, compared with the age group of 15 - 64 years. Hospital ownership explained the variation only in ICU accommodation and rehabilitation, while academic hospitals employed more surgical procedures and ventilation (Fig. 2).

In model 1, a one-point gain in the BI was significantly associated with shorter LOS and more TC. However, the interaction effect between the BI-at-admission categories with LOS and TC was observed to be significant in model 2 as a one-point gain in the BI was associated with shorter LOS in patients with dependent functional status and with more TC in those who were independent with assistance and nearly completely independent (Fig. 3).

\section{Discussion}

This study quantified the association of the magnitude of BI improvement with LOS and TC, using a community-based appraisal of patients with non-traumatic CVD in Japanese acute care hospitals. Better functional status at admission was observed to spare LOS and TC. BI improvement was observed to be independently associated with shorter LOS and more $\mathrm{TC}$, though we observed variation in incremental cost required for BI improvement among the BI-at-admission categories.

Outcomes like functional recovery (rather than mortality or complication) will receive greater attention from policy makers in this era of declining birthrate and aging population among the developed countries worldwide. To have a sustainable healthcare system, the elderly must maintain an active functional status. This is reason enough for healthcare service research to explore cost-effective treatment strategies, especially for first-ever stroke. In that situation, the BI has frequently been utilized as a risk adjustment factor. It has also been compared with other functional scales through profiling the content or criteria validity and through examin- 







\begin{tabular}{|c|c|c|c|c|c|c|c|c|}
\hline & \multicolumn{4}{|c|}{ LOS } & \multicolumn{4}{|c|}{ TC } \\
\hline & \multicolumn{2}{|r|}{ Model 1} & \multicolumn{2}{|r|}{ Model 2} & \multicolumn{2}{|r|}{ Model 1} & \multicolumn{2}{|r|}{ Model 2} \\
\hline \multicolumn{9}{|l|}{ Independent variables } \\
\hline Intercept & 11.0 & {$[6.3-15.7]$} & 11.1 & {$[6.4-15.8]$} & 154 & {$[-415-724]$} & 160 & {$[-410-731]$} \\
\hline \multicolumn{9}{|l|}{ Age (for $15-64$ years) } \\
\hline $65-74$ years & 0.3 & {$[-0.3-0.9]$} & 0.3 & {$[-0.3-0.9]$} & -80 & {$[-190-29]$} & -81 & {$[-190-28]$} \\
\hline $75-84$ years & -0.4 & {$[-1.0-0.2]$} & -0.3 & {$[-0.9-0.2]$} & -359 & \multicolumn{2}{|c|}{$[-471--246] \mid-358$} & {$[-470--246]$} \\
\hline 85 - years & -1.2 & {$[-2.1--0.4]$} & -1.2 & {$[-2.1--0.4]$} & -729 & {$[-894--565]$} & -728 & {$[-893--564]$} \\
\hline Male & -0.4 & {$[-0.9-0.0]$} & -0.4 & {$[-0.9-0.0]$} & 48 & {$[-40-135]$} & 47 & {$[-40-135]$} \\
\hline Destination at not at home & 6.3 & {$[5.7-7.0]$} & 6.3 & {$[5.7-7.0]$} & 302 & [182 - 422] & 302 & [182 - 422] \\
\hline Ambulance & 0.2 & {$[-0.3-0.7]$} & 0.2 & {$[-0.3-0.7]$} & 243 & [151 - 335] & 243 & [151 - 335] \\
\hline \multicolumn{9}{|l|}{ Principal diagnosis (for $\mathrm{T} \mid \mathrm{A}$ ) } \\
\hline Lacunar & 3.8 & {$[2.8-4.8]$} & 3.9 & {$[2.9-4.8]$} & 528 & {$[339-717]$} & 529 & {$[339-718]$} \\
\hline SAH & 1.8 & {$[0.2-3.5]$} & 1.9 & {$[0.2-3.6]$} & 8574 & \multicolumn{2}{|c|}{$[8256-8891]$ ] 8578} & {$[8260-8895]$} \\
\hline Heamorrhage & 6.5 & {$[5.3-7.6]$} & 6.5 & {$[5.4-7.6]$} & -1090 & \multirow{2}{*}{\multicolumn{2}{|c|}{$\begin{array}{l}\mid-1300--880] \mid-1089 \\
{[-6304--5686] \mid-5995}\end{array}$}} & {$[-1299--879]$} \\
\hline Subdural hematoma & -13.5 & {$[-15.2--11.9]$} & -13.5 & {$[-15.2--11.9]$} & -5995 & & & {$[-6304--5686]$} \\
\hline Infarction & 4.7 & {$[3.7-5.6]$} & 4.7 & {$[3.8-5.6]$} & 656 & [481 - 832] & 657 & {$[481$ - 832] } \\
\hline RIND & 3.5 & {$[2.1-4.9]$} & 3.5 & {$[2.1-5.0]$} & 705 & [439 - 970] & 707 & [441 - 972] \\
\hline \multicolumn{9}{|c|}{ Functional status at admission (for dependent) } \\
\hline Independent with assistance & 3.8 & {$[2.8-4.8]$} & 3.9 & {$[2.9-4.8]$} & 528 & {$[339-717]$} & 529 & {$[339-718]$} \\
\hline Nearly completely independent & -7.6 & {$[-8.5--6.7]$} & -8.5 & {$[-10.2--6.9]$} & 188 & \multirow[t]{2}{*}[22-354]{} & 203 & {$[-109-515]$} \\
\hline \multicolumn{8}{|l|}{ Charlson comorbidity index (for zero) } & \\
\hline 1 & 1.5 & {$[0.9-2.1]$} & 1.5 & {$[0.9-2.1]$} & 152 & {$[41-262]$} & 152 & [41 - 263] \\
\hline 2 & 2.3 & {$[1.6-3.0]$} & 2.3 & {$[1.6-3.0]$} & 162 & [33 - 292] & 162 & [33 - 292] \\
\hline 3 & 3.1 & {$[2.1-4.1]$} & 3.1 & {$[2.1-4.1]$} & 350 & [151 - 548] & 349 & {$[151-548]$} \\
\hline 4 & 4.0 & {$[2.1-5.8]$} & 4.0 & {$[2.1-5.8]$} & 105 & {$[-240-450]$} & 106 & {$[-239-451]$} \\
\hline 5 or more & 0.9 & {$[-2.3-4.0]$} & 0.9 & {$[-2.3-4.1]$} & 234 & {$[-373-840]$} & 238 & {$[-369-844]$} \\
\hline Cerebral vascular accident & -0.4 & {$[-1.1-0.3]$} & -0.4 & {$[-1.1-0.3]$} & 365 & [231 - 498] & 365 & [231-498] \\
\hline Atrial fibrillation or flutter & 1.0 & {$[0.1-1.9]$} & 1.0 & {$[0.1-1.9]$} & -101 & {$[-269-67]$} & -101 & {$[-269-67]$} \\
\hline Procedure related complication & -1.3 & {$[-4.1-1.6]$} & -1.3 & {$[-4.1-1.5]$} & 1121 & {$[588-1654]$} & 1121 & [588 - 1653] \\
\hline Deep vein thrombosis & 8.1 & {$[3.2-13.0]$} & 8.0 & {$[3.1-12.9]$} & 541 & {$[-396-1478]$} & 538 & {$[-399-1475]$} \\
\hline Cognitive impairment after admission & 3.4 & {$[-0.4-7.1]$} & 3.3 & {$[-0.4-7.1]$} & 1083 & {$[371$ - 1796] } & 1081 & [369 - 1794] \\
\hline Surgical procedure & 18.1 & {$[17.1-19.2]$} & 18.1 & {$[17.1-19.2]$} & 7417 & {$[7212-7622]$} & 7417 & [7212 - 7621] \\
\hline Administration in intensive care unit & -0.7 & {$[-1.5-0.1]$} & -0.7 & {$[-1.4-0.1]$} & 2886 & [2743-3029] & 2887 & [2744 - 3029] \\
\hline Ventilation & 12.0 & {$[9.6-14.5]$} & 12.0 & {$[9.6-14.5]$} & 6933 & {$[6469-7397]$} & 6932 & [6468 - 7396] \\
\hline Rehabilitation & 9.6 & {$[9.1-10.2]$} & 9.6 & {$[9.1-10.2]$} & 785 & [678 - 892] & 785 & [678 - 892] \\
\hline Hospital ownership (reference; national & & & & & & & & \\
\hline Municipal & 2.5 & {$[-2.4-7.5]$} & 2.5 & {$[-2.4-7.5]$} & -278 & {$[-842-285]$} & -278 & {$[-841-286]$} \\
\hline Non profit private & 3.2 & {$[-1.7-8.0]$} & 3.2 & {$[-1.7-8.0]$} & -109 & {$[-659-442]$} & -109 & {$[-659-442]$} \\
\hline Private for profit & 6.4 & {$[1.6-11.1]$} & 6.4 & {$[1.6-11.1]$} & 361 & {$[-181-903]$} & 361 & {$[-181-903]$} \\
\hline Hospital (for community) & & & & & & & & \\
\hline Academic & 1.1 & {$[-3.8-5.9]$} & 1.1 & {$[-3.8-6.0]$} & 1073 & {$[509-1637]$} & 1074 & [510 - 1638] \\
\hline Fiscal year (for 2009) & & & & & & & & \\
\hline 2008 & -3.7 & {$[-4.4--3.0]$} & -3.7 & {$[-4.4--3.0]$} & -25 & {$[-154-105]$} & -24 & {$[-154-105]$} \\
\hline 2007 & -2.3 & {$[-3.0--1.7]$} & -2.3 & {$[-3.0--1.7]$} & -174 & {$[-299--48]$} & -173 & {$[-298--47]$} \\
\hline 2006 & -1.4 & {$[-2.0--0.8]$} & -1.4 & {$[-2.0--0.8]$} & -545 & {$[-662--427]$} & -544 & {$[-662--427]$} \\
\hline Length of hospital stay & & & & & 302 & [299 - 304] & 302 & [299 - 304] \\
\hline Change in $\mathrm{Bl}$, one point gained & -0.031 & {$[-0.043--0.020]$} & ${ }^{a}-0.034$ & {$[-0.045--0.022]$} & 6 & {$[4.2-8.5]$} & a 3.9 & {$[-23.5-31.4]$} \\
\hline & & & ${ }^{\mathrm{b}} 0.004$ & {$[-0.042-0.050]$} & & & ${ }^{b} 9.2$ & {$[0.4-17.9]$} \\
\hline 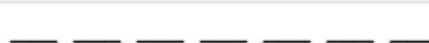 & & - & ${ }^{c} 0.053$ & {$[-0.091-0.197]$} & & & ${ }^{\circ} 6.2$ & {$[4.0-8.5]$} \\
\hline Akaike information criteria & & 189786 & & 189786 & & 420040 & & 420044 \\
\hline $\mathrm{Cl}$; confidence interval. RIND: reversib & emic & neurological de & it, SA & subarachnoid & $=\pi$ & age. Bl; Bar & & \\
\hline a; Dependent functional status at admis & on*chan & ge in $\mathrm{BI}, \mathrm{b}$; Inde & enden & with assistance at & t admi & sion*change & & \\
\hline c; Nearly complete independence at adr & sion*ch & hange in $\mathrm{BI}$. & & & & & & \\
\hline
\end{tabular}

Figure 3. Factors associated with length of hospital stay (LOS) and total charge (TC, US\$). 
ing the association or prediction with other relevant care-delivery outcomes such as patient destination or with resource utilization $[3,11,13,15,16]$. However, these investigations have often discussed the issue with the assumption that a one unit gain in functional scale score is weighted evenly among several kinds of functional status at admission. The BI (as well as other scales) was an ordinal variable scale originally and we felt that it was important to measure the contemporary validity of this functional scale on healthcare cost in acute care settings using the functional-status-at-admission categories. These fundamental estimations would surely contribute to predicting more precise healthcare costs for stroke care, which is the rationale for conducting this study.

Previous research has not always considered the economic impact of surgical procedures, critical care, or rehabilitation in acute care settings simultaneously, which has been proven to explain more of the variation in resource utilization by Kuwabara $\mathrm{K}$ et al $[5,21]$. In fact, Brahmbhatt $\mathrm{N}$ et al have emphasized the role of earlier mobilization in critical care settings to obtain better functional outcomes at discharge [22]. ICU care, ventilation, and rehabilitation should be enrolled in any model when assessing the economic burden during a care episode, as these care processes have been observed to increase costs. The next issue was whether these costly care processes were delivered to patients with worse functional status at admission. Surgical procedures, ICU care, ventilation, and rehabilitation were observed to be infrequently provided in the nearly completely independent patients. Japanese hospitals have therefore displayed an aggressive attitude to CVD care without neglecting patients with anticipated poor functional outcomes.

In considering the costs of care and the practice beliefs for CVD patients, the costs required for a one-point gain in the BI were varied in the mixed regression model. It has often been suggested that LOS should be shorter as earlier discharge with appropriate discharge arrangements is expected to optimize patient recovery [2]. The relationship between incremental cost and incremental functional recovery has not been quantified previously. Costs but not LOS were higher in the patients who were independent with assistance or nearly completely independent, and the impact between these two BI-at-admission categories was different. Costs were insignificantly different in patients with a dependent functional status at admission, though the $95 \%$ confidence interval was wider, compared with the other two categories. Although we have attempted to partly take into account the effect of ventilation administration as a proxy for worse consciousness level, patients groups with a dependent functional status at admission might have included more than two case-mixes, which might be, for example, either patients with stable spontaneous breathing and worse consciousness level or patient case-mixes requiring more costs unexplained by the patient- or care-related variables. Consciousness level by the Japanese coma scale both at admission and discharge, though not in use worldwide, has been gathered in this administrative database and will provide a promising research topic for minimizing unexplained variation in costs.

In this analysis, not every patient in the advanced age categories consumed more LOS or TC compared with patients aged $15-64$ years $[6,10]$. However, the baby-boomer generation will surely carry more weight as the stakes and the economic burdens of healthcare increase. Healthcare service researchers or policy makers should also recognize the inherent variation in functional scales like the BI in this study when assessing the economic analysis of CVD-related care and estimating healthcare costs, but not LOS [1]. When formulating healthcare policy for CVD patients to promote functional convalescence, they should implement effective policies supported by evidence that includes functional status category at admission.

We should mention several limitations of this study. First, data were collected from discharged patients from hospitals voluntarily participating over four consecutive years. This limits the generalizability of our results. However, the MHLW have gathered exhaustive data and is now extending the data collection period to 12 months, which will overcome this limitation.

Second, this study lacked information regarding the severity of cognitive impairment, the onset time of CVD, and other motor or cognitive scales that are often utilized in health service research [4]. The Japanese Stroke Databank (JSD) has separately collected such clinically informative data and we may be able to obtain more meaningful findings using the administrative database in combination with the JSD [23]. Since 2010, the MHLW have started to collect another functional scale (mRS) and the stroke onset time, which would allow refinement of a study methodology and our results because functional recovery is expected to be dependent on the post-stroke times between stroke onset and treatment.

Third, the intensity of rehabilitation in acute care settings was not considered, but units of rehabilitation delivered might be collected in our database. Meanwhile, the mean LOS for acute care hospital admissions has decreased from 24.8 days in 2000 to 19.2 in 2006 , but was still 2 - 4 times longer than that in several Organization for Economic Co-operation and Development (OECD) countries in 2006 [24]. The OECD acknowledged that the reason for the longer LOS was partly because of the broader differentiation of "acute care" compared with the definition used in other OECD countries [25]. Ironically, this kind of study is then made more possible and earlier rehabilitation and functional outcomes could be counted more precisely than in western countries with shorter LOS.

\section{Conclusion}

We quantified the association of improvement in the BI score 
with cost, based on a community-based study of the CVD patients in Japanese acute care hospitals. Care was delivered to the more disabled patients at admission. Though better functional status at admission was observed to decrease LOS and TC, and BI score improvement was independently associated with shorter LOS and more TC, we observed a variation in incremental cost required for BI improvement among the various functional status groups at admission. To plan effective and efficient healthcare policy for CVD patients for promoting functional convalescence, the variation in cost required for a given functional gain depending on the functional status category at admission must be incorporated.

\section{Grant Support}

This study was funded in part by Grants-in-Aid for Research on Policy Planning and Evaluation (Japanese Ministry of Health, Labor and Welfare, H19 seisaku-sitei 001).

\section{References}

1. Ministry of Health, Labor and Welfare Authority. National Medical Care Expenditure (Estimates). http:// www.mhlw.go.jp/shingi/2006/09/s0906-6.html Access March 282011.

2. De Wit L, Putman K, Schuback B, Komarek A, Angst F, Baert I, Berman P, et al. Motor and functional recovery after stroke: a comparison of 4 European rehabilitation centers. Stroke. 2007;38(7):2101-2107.

3. Lauretani F, Saccavini M, Zaccaria B, Agosti M, Zampolini M, Franceschini M. Rehabilitation in patients affected by different types of stroke. A one-year follow-up study. Eur J Phys Rehabil Med. 2010;46(4):511-516.

4. Pereira SR, Chiu W, Turner A, Chevalier S, Joseph L, Huang AR, Morais JA. How can we improve targeting of frail elderly patients to a geriatric day-hospital rehabilitation program? BMC Geriatr. 2010;10:82.

5. Kuwabara K, Matsuda S, Fushimi K, Ishikawa KB, Horiguchi H, Fujimori K. Reconsidering the value of rehabilitation for patients with cerebrovascular disease in Japanese acute health care hospitals. Value Health. 2011;14(1):166-176.

6. Kugler C, Altenhoner T, Lochner P, Ferbert A. Does age influence early recovery from ischemic stroke? A study from the Hessian Stroke Data Bank. J Neurol. 2003;250(6):676-681.

7. Yoneda Y, Okuda S, Hamada R, Toyota A, Gotoh J, Watanabe M, Okada Y, et al. Hospital cost of ischemic stroke and intracerebral hemorrhage in Japanese stroke centers. Health Policy. 2005;73(2):202-211.

8. Di Carlo A, Lamassa M, Baldereschi M, Pracucci G, Consoli D, Wolfe CD, Giroud M, et al. Risk factors and outcome of subtypes of ischemic stroke. Data from a multicenter multinational hospital-based registry. The European Community Stroke Project. J Neurol Sci. 2006;244(1-2):143-150.

9. Appelros P. Prediction of length of stay for stroke patients. Acta Neurol Scand. 2007;116(1):15-19.

10. Saposnik G, Black SE, Hakim A, Fang J, Tu JV, Kapral MK. Age disparities in stroke quality of care and delivery of health services. Stroke. 2009;40(10):3328-3335.

11. Dawson J, Lees JS, Chang TP, Walters MR, Ali M, Davis SM, Diener HC, et al. Association between disability measures and healthcare costs after initial treatment for acute stroke. Stroke. 2007;38(6):1893-1898.

12. Epifanov Y, Dodel R, Haacke C, Schaeg M, Schoffski $\mathrm{O}$, Hennerici M, Back T. Costs of acute stroke care on regular neurological wards: a comparison with stroke unit setting. Health Policy. 2007;81(2-3):339-349.

13. Giles LC, Hawthorne G, Crotty M. Health-related Quality of Life among hospitalized older people awaiting residential aged care. Health Qual Life Outcomes. 2009;7:71.

14. Sulter G, Steen C, De Keyser J. Use of the Barthel index and modified Rankin scale in acute stroke trials. Stroke. 1999;30(8):1538-1541.

15. Lin JH, Hsu MJ, Hsu HW, Wu HC, Hsieh CL. Psychometric comparisons of 3 functional ambulation measures for patients with stroke. Stroke. 2010;41(9):2021-2025.

16. Banks JL, Marotta CA. Outcomes validity and reliability of the modified Rankin scale: implications for stroke clinical trials: a literature review and synthesis. Stroke. 2007;38(3):1091-1096.

17. Matsuda S. Casemix as a tool for transparency of medical services. Jpn J Soc Sec Policy. 2007; 6: 43-53.

18. Sundararajan V, Henderson T, Perry C, Muggivan A, Quan H, Ghali WA. New ICD-10 version of the Charlson comorbidity index predicted in-hospital mortality. J Clin Epidemiol. 2004;57(12):1288-1294.

19. Zhan C, Miller MR. Administrative data based patient safety research: a critical review. Qual Saf Health Care. 2002; 12: 58-63.

20. Hayashida K, Imanaka Y, Otsubo T, Kuwabara K, Ishikawa KB, Fushimi K, Hashimoto H, et al. Development and analysis of a nationwide cost database of acute-care hospitals in Japan. J Eval Clin Pract. 2009;15(4):626633.

21. Kuwabara K, Imanaka Y, Matsuda S, Fushimi K, Hashimoto $\mathrm{H}$, Ishikawa KB, Horiguchi H. Profiling of resource use variation among six diseases treated at 82 Japanese special functioning hospitals, based on administrative data. Health Policy. 2006;78(2-3):306-318.

22. Brahmbhatt N, Murugan R, Milbrandt EB. Early mobilization improves functional outcomes in critically ill patients. Crit Care. 2010;14(5):321.

23. Kobayashi S. International experience in stroke registry: 
Japanese Stroke Databank. Am J Prev Med. 2006;31(6 Suppl 2):S240-242.

24. Organization for Economic Co-operation and Development (OECD). Health Data 2008 - Frequently Requested Data. http://www.irdes.fr/EcoSante/DownLoad/
OECDHealthData_FrequentlyRequestedData.xls Access March 282011.

25. Organization for Economic Co-operation and Development (OECD). Health at a Glance OECD indicators 2005. Paris: OECD Publishing; 2005: 56. 